1965

\title{
COMPLEMENTARITY AND THE SCIENTIFIC METHOD: A CRITICISM
}

Patrick A. Heelan

Georgetown University, heelanp@georgetown.edu

Follow this and additional works at: https://fordham.bepress.com/phil_research

Part of the Continental Philosophy Commons, History of Philosophy Commons, and the Philosophy of Science Commons

\section{Recommended Citation}

Heelan, Patrick A., "COMPLEMENTARITY AND THE SCIENTIFIC METHOD: A CRITICISM" (1965). Research Resources. 27. https://fordham.bepress.com/phil_research/27

This Article is brought to you for free and open access by the Hermeneutic and Phenomenological Philosophies of Science at DigitalResearch@Fordham. It has been accepted for inclusion in Research Resources by an authorized administrator of DigitalResearch@Fordham. For more information, please contact considine@fordham.edu. 
CHAPTER FOUR

COMPLEMENTARITY

AND THE SCIENTIFIC METHOD: A CRITICISM

SECTION I: PROPOSITION (I) ON SCIENTIFIC METHOD

\section{Proposition (I)}

The definition of quantum mechanical variables can only be made with the aid of classical physical concepts. These are identical except for refinements - with the concepts of everyday life. Heisenberg has written: "The concepts of classical physics will remain the basis of any exact and objective science. Because we demand of the results of science that they can be objectively proved (i.e. by measurements, registered on suitable apparatus) we are forced to express these results in the language of classical physics... Thus while the laws of classical physics. . appear only as limiting cases of more general and abstract connections, the concepts associated with these laws remain an indispensable part of the language of science without which it would not be possible even to speak of scientific results" 1.

\section{Criticism}

We contend that there are two logically distinct sets of concepts in physics, whether in classical or in quantum physics; and that failure to advert to this vitiates the above proposition. We attribute such failure to a theory of knowledge implicit in the philosophy of complementarity, a theory which is usually given the name psycho-physical parallelism. As psycho-physical parallelism is the key to, the philosophic thinking of many physicists to-day, we shall devote the following paragraphs to it.

Psycho-physical Parallelism

The intentionality-structure of classical physics implied a naive Cartesian Dualism of Mind and Body. In its original fonn, Cartesian author's Physics and Philosophy (New York: Harper, 1958), pp. 44, 144. 
Dualism regarded the knowing subject as "mirroring" the known object (its referent), and the accuracy of the representation was guaranteed by the veracity of God. Under the influence of the Kantian critique, this dualism was transformed into a parallelism and was introduced into the interpretation of modern physics by Bohr 1. His psychophysical parallelism postulates a unique "translation" of physical events into psychic acts of observation (called "sensations") 2. Science, then, concerns itself with the organisation of these "sensations" on the empirical level, and with the construction of theoretical entities (theoretical constructs) to give them a coherent ground in a unified consciousness. Whether the "sensations" were given an empiricist or idealistic interpretation, the core idea was the same, namely, an isomorphism between the content of conscious acts of observation (the sensations) and the unconscious physical events which they express and which are their underlying cause. The function of mind called sensation models physical events - not in the naive realistic way of Cartesian Dualism which was based upon the isomorphism of (idealised) bodies imaginatively represented with the external world - but only in observation events (Beobachtungsvorgange). Only observation-events express knowledge of physical reality. Physical reality is, by definition, expressed by the type of sensations called "observation-events". A scientific observation-event is one accomplished with the aid of instruments.

Implicit in this view are two propositions about the nature of scientific method: (a) a physical property is the $\backslash$ direct physical correlate of the empirical content of a scientific observation-event. In other words, the act of observation translates the appropriate physical property into the empirical content of a conscious act; that is, just as "physical colour" is related to "colour-as-sensed", and "physical shape" is related to "shape-as-seen", so a "physical property" is related to the "property-as-observed in the scientific observation-event". This

1 The Principle of Psycho-physical Parallelism was first expressed by G. Th. Fechner, and it influenced the interpretation of physics through Wilhelm Ostwald's Lectures in Natural Philosophy. Extracts from Ostwald's lectures are included in Heisenberg's collection: "The Origins of the Mechanistic and Materialist World-View", Physicist's Conception of Nature, pp. 137-151. (Although Heisenberg's name is on the book-cover, it is not clear that he was also the compiler of the extracts. The extracts at any rate have a value of their own).

2 N. Bohr, Naturwissen., XVII (1929); translated and published under the title "The Atomic Theory and the Fundamental Principles of the Description of Nature", in Atomic Theory and the Description of Nature, pp. 102-119. The first systematic use of the principle in the quantum theory of measurement was made by $\mathbf{J}$. von Neumann in Mathematische Grundlagen der Quantenmechanik, (Berlin: Springer. 1932). In the English trans. by R. T. Beyer, Mathematical Foundations of Quantum Mechanics, it is stated on p. 420. 
implies that there is no logical difference in structure between the way we express a physical property in a scientific concept and the way we express an everyday property in an everyday concept. (b) Between the use of everyday (pre-scientific) concepts and the use of scientific concepts there is the difference that in science the intervention of instruments occurs. These enable publicly objective comparisons to be made and verified by different people and add a new exactness of expression to the concepts through the use of mathematical relationships. They also perform the functions of filtering out unwanted elements in a complex situation, of magnifying those we want, or of "translating" them into new forms which our powers of perception can better recognise and deal with. Useful and in fact indispensable as these instruments are in physics, this view holds that they really come between the physicist and the physical object, and, if the physical object is very small, the presence of the instrument disturbs it. According to this theory, which we called the perturbation theory of measurement, the principal discovery of quantum mechanics is the essential limitation of physical instruments to reveal very small objects as they really are.

The second assertion (b) above will be considered fully in the next section. In reply to the first assertion (a), we distinguish between two classes of concepts. One has a logical structure based upon the resemblances of things as regards their appearance to us and the uses they have in the practical affairs of life. These are descriptive concepts, based upon thing-to-us relations, and they describe a World of things and properties which are for-us. These are the concepts of everyday life, enlarged and specialised so as to be able to describe experimental procedures (operational concepts) and experimental results (observational concepts).

The other class of concepts has a logical structure based upon resemblances as regards the mutual interactivity of things. They are explanatory concepts, founded upon thing-to-thing relations and they describe a World of things for-things and of properties for-things, notably for that class of things which can serve our purpose as measuring instruments 1 . The resemblances on which these concepts are based

1 The expression World-for-things does not connote that an observer-instrument (which is the thing in the World-for-things) has a consciousness like a human observer open to a horizon of reality called a World. Reality is known only by a human observer. But just as everyday realities are known within a World-structure which is a set of relations to the knower, so we state that scientific realities are known within a World-context of a set of relations centered on things (or observer-instruments). One might take the expression "World-forthings" to be shorthand for "World-of-things-to-things-for-us". See below, chapter VII. 
cannot be known directly, since they do not concern how things look or appear to us. They can only be inferred from their effects in nature and in the controlled environment of the laboratory. These effects are sensible signs or observable symbols which, when interpreted correctly, reveal a network of related activities among things. Since a physical theory is the expression of an insight into such a set of interrelated activities, an act of observation is not simply the translation into consciousness of a physical event. It is the perception of a sensible event and, with it, the recognition that it is endowed with a symbolism revealing something which is not per se sensible, since it is neither a thing-for-us nor a property-for-us, but a thing-for-things or a propertyfor-things. As a consequence, the function of a measuring instrument which is the term of such relations - is not just to bring a degree of exactness and public objectivity into science which everyday knowledge lacks, but to help to create a new kind of knowledge, based upon a new kind of concept, expressing a new kind of relation. Just as the observed effects of a physical property are known only through observable symbols, like pointer readings, etc., so the mind can deal with these properties only through a constructed mathematical symbolism which symbolises in turn these observed effects and which, through its mathematical form, reveals the essential, relational structure of the symbolised properties.

A physical property or variable, then, is expressed by us as the union of two concepts - an explanatory concept 1 and an operational concept. The explanatory concept draws its intelligibility from a systematic totality which in the concrete is a sphere of reality to which we give the name World-for-things and is the sphere of the scientific real. Operational concepts and observational concepts draw their intelligibility from a systematic totality which in the concrete is the sphere of the everyday real which is called the World-for-us. Each World has its own symbolic embodiment in a language; the observation language of the World-for-us and the explanatory language of the World-forthings, which are linked by their common denotation 2. The linguistic link between the two languages is called a correspondence rule.

We stress the operational aspect of physical properties, since it is by certain activities on our part that we produce the controlled environment in which things interact among themselves in such a way as to ground a single thing-to-thing relation and a single property. But we 
do not say, as Bridgman and the advocates of operationalism say, that the physical concept is no more than a generalised set of procedures to be performed by us. The physical concept explains the procedures, that is, it gives the reason why they measure one single property (and not a mixture of properties). It also explains why the set of measuring devices for a single property is an open set.

\section{A Pseudo-problem}

The distinctions we have just made belong to physical (or scientific) method in so far as this is a human way of investigating reality. From the failure to distinguish the human element in physics from its proper object, many pseudo-scientific problems arise. Complementarity, for instance, fails to recognise the difference in logical structure between physical concepts and the concepts of everyday life, and consequently overlooks the difference between the observable symbol which is an event in everyday life and the physical thing or property which is essentially unrepresentable in observational concepts. This gives rise to a series of pseudo-scientific problems based upon the dilemma: Is a quantum system a wave or a particle? In chapter $v$ we explain our reasons for stating that it is at the same time both a wave and a particle, and neither one nor the other. It is neither, since a particle and a wave as objects of observation belong to the symbolic order and do not constitute the reality of the quantum system; it is both a wave and a particle, however, since "wave" and "particle" describe aspects of the mathematical formalism within which, in some way, the constitution of the quantum system is defined.

\section{Conclusion}

To summarize briefly the content of this section: it is our view that classical and quantum physics share the same operational and observable concepts, but that they differ in explanatory concepts. We shall return to this point in a later chapter.

We have also deduced that observable physical data have a two-fold reality: the physical reality of an observable symbol (e.g., a pointer reading, etc.), and the intentional reality of a property symbolised. The direct empirical object of the act of observation is the sensible symbol; the indirect object (known only through interpretation) is the property symbolised. The mathenlatical expression of a scientific theory parallels this twofold reality by using mathematical symbols in two ways, (a) to define the properties by implicit definition, and (b) to interpret the 
mathematical symbol observationally, i.e., by indicating what observable symbols (for example, the reading of a dial or the average of such readings), the mathematical symbol stands for.

The failure to distinguish between observable symbol and physical property can lead to a variety of philosophical opinions about the representative value of science. All fonus of parallelism, for instance, lead to the fallacious view that the aim of scientific method is to construct, if possible, a perceptible (anschaulich) model of reality directly "translatable" into ontological terms. By "perceptible model" we mean in general, one constructed on the basis of sensible thing-tous relations. Such a model may be thought of as a true model expressing reality-as-it-is-in-itself (realism), or merely as a surrogate model useful merely for prediction and practical purposes (instrumentalism).

With regard to the ontological value of physics, physicists, roughly speaking, take up one of two positions. The first is a rationalist realism for macrophysics following the tradition of classical physics. This is often accompanied by a rationalist instrumentalism in the field of quantum physics, because of the failure of quantum physics to construct a precise model based upon the classical limiting concepts of particle and field. This is the position, for example, of Einstein, Schrodinger, Bohm, Vigier, Rosen, et al. It is especially characteristic of physicists who have specialised in the theory of relativity and are looking for a unitary field theoretic description of the universe. What characterises this group is a Platonic tendency to equate the meaning of reality with whatever can be understood conceptually, leaving empirical experience merely to provide the occasion for the recognition of existence. The second is an empiricist realism with regard tomacrophenomena. This conceives physical reality to be no more then what is given factually in experience. This is often accompanied by an empiricist or positivistic instrumentalism in quantum physics, because the abstract norms of quantum physics, as, for example, the Psi-function, are so unanschaulich or unimaginable 1 . We shall return to these distinctions in a later chapter.

\section{SECTION II: PROPOSITION (2) ON SCIENTIFIC METHOD}

\section{Proposition (2)}

The act of measurement perturbs the object. Its objective state ("objective", that is, "not affected by the subjectivity of purely private 
experience") then cannot be known - whether as an object of empirical science (a phenomenal object) or as a reality (an object in the strict or formal sense) 1. The Indeterminacy Principle expresses the degree of this perturbation, and thereby traces the limits of our power of knowing the physical object. Heisenberg has written: "Our ordinary description of nature, and the idea of exact laws, rests on the assumption that it is possible to observe the phenomena without appreciably influencing them. To co-ordinate a definite cause to a definite effect has sense only when both can be observed without introducing a foreign element disturbing their interrelation. The law of causality, because of its very nature, can only be defined for isolated systems, and in atomic physics even approximately isolated systems cannot be observed... for in atomic physics we are dealing with entities that are (so far as we know) ultimate and indivisible. There exist no infinitesimals by the aid of which an observation might be made without appreciable perturbation" 2. On the Indeterminacy Principle, he says that it "refers to the degree of indeterminateness in the possible present knowledge of the simultaneous values of various quantities with which the quantum theory deals" 3.

\section{General Criticism}

The perturbation theory of measurement implies that the activities which take place between object and instrument in the measuring process serve no other function than to render some physical system or some property of it accessible to a human observer by magnifying it, otherwise "translating" it into a form in which it can produce a perceptible impression on a human observer. The measuring process is accused of perturbing to a greater or lesser extent the real physical property which one wishes to measure. This theory implies that the real physical property is other than what is defined by the measuring process itself. It also implies that a physical property is a thing-to-us relation. Two opinions are worth noting: (a) that of Einstein, Podolsky and Rosen who would define a physical property as what is left after the disturbance is removed 4 , and (b) that of Bohr, Heisenberg and others who would say that the disturbance is not removable either in

sophical outlooks of relativists and quantum theorists, see E. Wigner, "Relativistic Invariance and Quantum Phenomena", Rev. Mod. Phys., Xxix (1959), pp. 255-268.

1 The objectivity here denied is "public objectivity", a type of objectivity which has always been regarded as a characteristic property of scientific knowledge. Cf. chapter v.

2 Heisenberg, Physical Principles etc., pp. 62-63.

3 Ibid., p. 20.

4 Phys. Rev., XLVII (1935), p. 777. 
fact or in principle, thus implying that all that can be known is an interaction in which subject and object are inextricably mixed. This latter position (b) leads to a distinction between physical states and physical properties: a physical state being a set of relations to possible observers which do not, however, define the physical properties of the systenl while a physical property is the inaccessible residue of what would be left if the disturbance could be removed. We believe that the rational outcome of this dialectic is to affirm that a property of a physical system is defined by its relations to other systems within the measuring process, and not with respect to the possible direct perceptible experience of a human observer. We put forward, then, a relational theory of physical properties 1 .

\section{The Relational Structure of Physical Properties}

It is our view that a physical property (or a property of a physical system) is the term of a relation set up between the physical system and a measuring instrument: a relation resulting from the production of a formal or proper effect in the measuring instrument by the interaction during the measuring process. This proper or formal effect may not itself be a sensible datum. It may be, for example, the emission of a single photon and this is below the threshold of sensitivity. The proper effect, however, must be such that it can be subsequently transformed into a sensible datum through magnification techniques, like the use of micro-ammeters, scintillation counters, etc. It is the outcome of such a transformation that we termed an observable symbol.

The action between the object and the instrument is an interaction. There is, consequently, an effect produced in each of the interacting terms. The theory allows each of these effects to be used to measure the same property in the other member of the interacting pair. For example, the recording of a photon of energy $\mathrm{E}$ may be witness of an exothermic radioactive disintegration of $\mathrm{Q}$-value equal to $\mathrm{E}$, or, conversely, the recording of the energy of the disintegration fragments could be used as witness that a photon of energy E was emitted. Every

1 Similar ideas have been put forward by P. K. Feyerabend in "Problems of Microphysics", Frontiers of Science and Philosophy, ed. by R. G. Colodny (London, Allen and Unwin, 1963), pp. 189-283, and by M. Sachs in "A New Approach to the Theory of Fundamental Processes", Brit. Jour. Phil. Sci., xv (1964),PP. 213-243. Sachs formulates the principle that the laws of Nature must be described in terms of field variables that may be associated only with elementary interactions (p. 221 our italics). Weyl seems to hold a similar position, as for example, in Gruppentheorie und Quantenmechanik, p. 66, but the passage is not reproduced in the English translation. It is clear, however, from Appendix C of his Philosophy ot Mathematics and Natural Science, pp. 253-265, that he too adheres essentially to the perturbation theory of measurement, though he may not have been a ware of all its logical consequences. 
well-designed measuring-process, then, has a structure which can be represented by the formula, $\overleftrightarrow{\mathrm{aPb}}$, where $\stackrel{\leftrightarrow}{\mathrm{P}}$ represents the interaction characteristic of the property $\mathrm{P}$, and $\mathrm{a}$ and $\mathrm{b}$ are the terms affected and so correlated by the interaction. It follows then that $\mathrm{P}$ founds a

twofold relation: (1) $\mathrm{aPb}$, which reads: "The formal effect of $\mathrm{P}$ on the instrument $b$, enters into the definition and measurement of the property $\mathrm{P}$ of the object a". (2) aPb, which reads: "The formal effect of $\mathrm{P}$ on the instrument a enters into the definition and measurement of the property $\mathrm{P}$ of the object $\mathrm{b}$ ".

Not every interaction between $a$ and $b$ is or could become $a$ measuring-process. A necessary condition is that such an interaction should be simple, i.e., that a virtually single formal effect should be produced in a and in $\mathrm{b}$ (or if the formal effects be multiple, that all but one could be filtered out). How is one to know which interactions are potential measuring-processes and which are not? This is known not by empirical generalisation from many cases, but by interpreting the experimental process with the aid of a physical theory. It is on the authority of a physical theory (or hypothesis) that such and such an interaction is declared (positively or hypothetically) to be simple.

Each of the relations $\overrightarrow{\mathrm{aPb}}$ and $\overleftarrow{\mathrm{aPb}}$ is founded upon an absolute ground in a and in b respectively. So far as our knowledge goes, the physical property is the absolute ground which orients the physical system to the production of an appropriate formal effect in other things. We call the absolute ground the primary relativity of a physical property. What then is the absolute ground?

We have already mentioned that a definition defines not a concrete essence in its particularity, but an ideal norm, and an ideal norm expresses a certain similarity in which many things (actually or potentially) share. The similarity in question here is a similarity in the way things act upon one another as, for example, in the two relations $\overrightarrow{\mathrm{aPb}}$ an $\mathrm{aPb}$. The property, as so defined, is an explanatory property. It follows from this that we only know the properties of things within a pattern of relations which is itself grounded upon a pattern of interactions. It might be surmised that another kind of intellect would be capable of knowing the ground of a property absolutely. Some might say even that the human intellect in other non-scientific kinds of knowledge 
would be able to know the ground of a property absolutely. However, even if this were so, it might still be doubted whether the absolute ground for any of the relations we are talking about would turn out to be intelligible apart from a World of actually related and interacting things which would give meaning to the ground. It is our view that the essential nature of the ground is to be oriented towards action with and upon things and sQ to the constitution of a World.

The pattern of relations which define the explanatory concepts is the physical theory. Let the relations which found the physical properties be symbolised by $\mathrm{Pi}(\mathrm{i}=1,2, \ldots, \mathrm{n})$; let $\mathrm{Pi}(\mathrm{i}=1,2, \ldots, \mathrm{n})$ be the corresponding interactions, and let $\mathrm{Pi}(\mathrm{i}=1,2, \ldots, \mathrm{n})$ be a numerical variable obtained by mapping the formal effects onto the real number field by a system of meters, circuits, etc. The mapping may be done in either of two ways: either by a direct mapping of individual concrete experiments onto the number field - this is the way of quantum mechanics; or by an indirect mapping whereby individual concrete values are taken as samples of some abstract ideal value (for example, an average value) - this is the way of classical physics 1. Whatever manner of mapping is used, the physical theory asserts a certain set of equations:

$$
f_{j}\left(p_{i}\right)=0, \quad j=1,2, \ldots, m
$$

These equations have the effect of defining $\mathrm{Pi}$ in a mutual fashion by implicit definition of the set of variables pi; in other words, we say that the set $\mathrm{Pi}$ is a self-defining set of numerical variables. As the $\mathrm{Pi}$ are uniquely detern1ined with respect to the formal effects of $\mathrm{Pi}$ (through the magnification or other transformation which produces the respective observable symbol), the implicit definition of the Pi can be interpreted to mean that the set $\{\mathrm{Pi}\}$ of the physical interactivities is a self-correlated set; or, in other words, that the set of relations $\{\mathrm{Pi}\}$ is a closed self-defining set of relations.

In quantum mechanics, the properties are represented by linear operators $\mathrm{Pi}$ and not by numerical variables. The equations of the axiomatic theory are operator equations on a Hilbert space of physical states. Each operator $\mathrm{Pi}$ represents a physical activity $\mathrm{Pi}$, and its eigen values are the possible range of values Pi. As in the former case, the operator equations have the effect of mutually defining Pi by implicit definition of the set $\{\mathrm{Pi}\}$. This implicit definition of Pi can 
be interpreted to mean that the set $\{\mathrm{Pi}\}$ of physical activities is a selfcorrelated set and that the set of relations $\{\mathrm{Pi}\}$ is a self-defining set. A pair of non-commuting operators would mean that the physical activities corresponding to these operators interfere with one another in the concrete. We shall postpone further discussion of the various interpretations of the quantum mechanical formalism to another section. For the present, it is sufficient if we have made it clear what we mean when we say that a physical property is the term of a relation founded upon interactivity; that its primary relativity is defined implicitly by an explanatory definition which involves a systematic totality constituted by a mutually-defining set of interrelated properties 1 .

Returning to the act of measurement: this is completed by an act of observation in which the observer-scientist recognises certain sensible data either as the formal effect of a certain property $\mathrm{Pi}$ of the measured object, or as something uniquely derived from it through ancillary devices, such as meters, circuits, etc. Besides providing instances of a physical property $\mathrm{Pi}$, the sensible data also provide the values of the secondary detenninations associated with the measured property. These are the measure-numbers $\mathrm{Pi}$ of the property.

In classical physics, these measure-numbe are treated in either of two ways. (I) Abstractly - as samples of an idealised model of a physical process; this treatment leads to a deterministic theory like Newtonian mechanics. Consequently, the properties of classical physics are affected by a certain ideal and abstract character which is intrinsic to the method used. Or (2), they may be used as a set of individual values, in which case they constitute a statistical distribution (of the type of a "distribution of errors") of which the ideal classical model is the mean. A characteristic of classical physics is that a statistical theory is distinct from the deterministic theories which define the elements of the statistical ensemble.

Quantum mechanics differs from both of the older types of physical theories. It has in common with both, however, the common structure of human scientific knowing. Quantum mechanics, then, expresses both

\footnotetext{
1 "The concepts with which natural science deals are not qualities or attributes which can be obtained from the objective world by direct cognition. They can only be obtained by an indirect methodology, by observing their reactions with other bodies, and their implicit definition is consequently conditioned by definite laws of nature governing reactions", H. Weyl, Theory of Groups and Quantum Theory, trans. by H. P. Robertson (New York: 1931), p. 76; d. also his PhilosoPhy of Math. etc., pp. 137-164. For Weyl, however, the concept does not express what is intrinsic to the physical object. For an example of how a physical theory like Newtonian Mechanics is composed of undefined elements defined implicitly by mathematical operations, ct., P. Suppes, Introduction to Logic (Princeton: 1957), pp. 291-304.
} 
an idealised model and, at the same time, tries to make allowance for the variety of concrete cases. This makes the problem of separation method and object in quantum mechanics more difficult than in the older theories, since it seems to have two objects and two methods which, according to the majority of physicists, are inseparable from one another. On the other hand, quantum mechanics has a kind of simplicity which neither of the older physical theories possesses; it is concerned with the concrete instances of the ideal model or norm (or, alternatively, the ideal law in its concrete instances) in their simultaneous and actual union. Quantum mechanics is concerned, as Heisenberg has said, with observation-events, that is with physical reality in the most immediate and actual form in which it presents itself to an investigator.

A characteristic feature of a classical theory is that the six state variables of each particle, i.e., the three of position and the three of momentum, are at each instant, independent degrees of freedom each with a determinate value. Quantum physics overthrows this assunlption. It shows that the measure-numbers for the six state variables are not independent in the concrete, and, consequently, that position and momentum do not constitute for the individual concrete particle six independent degrees of freedom.

\section{Remnants of Classical Rationalism}

Why should this discovery have shocked physicists so much? The reason was that, when quantum mechanics was discovered, physicists had long been accustomed to accept uncritically the rationalist outlook on physical reality characteristic of classical physics. If physical reality is the subject of a classical description, then physical reality is something ideal and abstract, viz., the content of a conceptual definition. Quantum mechanics showed that concrete reality, as manifested in empirical data, is capable of no such definition. Position and momentum are concretely correlated variables and not independent (aspects of a perfect conceptual model). Either physical reality was parallel to a perfect conceptual model but was unknowable, or else physical reality was known only in the concrete data. The first impact of quantum mechanics was to send science back to individual concrete experience. Science must return to the concrete, i.e., to the instances of physical reality revealed in observation events. Many elements of rationalism remained, however, in the revised outlook, of which the perturbation theory of nleasurement is a good example. In spite of the 
conversion to a basic ontological empiricism, as to what gives meaning to physical reality, the classical notion of a perfect set of measurenumbers tended to remain as the criterion which the physical reality must satisfy. The perturbation theory of measurement witnesses to the continuation of a strong current of rationalism within Heisenberg's view of complementarity. This will be discussed in chapter VIII.

We hold, on the contrary, that the perturbation which takes place when two conjugate properties are measured is a new revelation of the properties of nature, and that this has led to a more accurate definition of them, which now includes this perturbation as an essential part. Thus, the Indeterminacy Relations supply not less, but more, information about the object of physics then was possible before, since, in addition to describing the kinds of similarities that exist between things, it also tells us how intimately some are related to others in concrete individual cases.

\section{The Indeterminacy Relation}

In our interpretation of the Indeterminacy Relation (or Indeterminacy Principle) we agree with Heisenberg in the following points: (a) that it expresses the fact that concrete acts of measuring conjugate variables generally and regularly interfere with one another 1; (b) that it is in some way a measure of this mutual interference, and (c) that it can be interpreted in two ways: as applicable to individual systems or as applicable to ensembles of identical systems.,

The Indeterminacy Principle for individual systems is expressed by the non-commutation of conjugate operators 2 ; for example, of $\mathrm{x}$ (position) and $\mathrm{p}$ (the conjugate momentum). An operator represents a property of an individual system. A property, as we have said, is related to the act of measurement. Hence, the Indeterminacy Principle states something about the incompatibility of conjugate properties of an individual system even before an actual measurement is made 3.

The Indeterminacy Principle for ensembles of identical systems is expressed as the lower limit of the product of two standard deviations, e.g., $\overline{\mathrm{Dx}} \cdot \overline{\mathrm{Dp}} \geq \mathrm{h}$ 4. In this form, it is a statistical principle, and belongs properly only to ensembles of identical systems considered with respect to the possible outcome of measurements made on each.

1 Heisenberg, Physical Principles etc., p. 3 and passim.

2 Ibid., pp. 118-123.

3 Ibid., pp. 13-14, 20-33.

1 Ibid., pp. 15-19, 34-46. 
However, this does not exhaust the problem, for we can inquire further whether, and in what sense, the Indetenninacy Principle states that the "use of the words 'position' and 'velocity' with an accuracy exceeding that given [by this principle] is just as meaningless as the use of words whose sense is not defined" 1 . Heisenberg's answer is that, since $\mathrm{p}$ and $\mathrm{x}$ are conditioned by their respective measuring-processes, a non-compatibility of conjugate measuring processes leaves the simultaneous pair $(\mathrm{p}, \mathrm{x})$ unrealisable 2 ; that is to say, it is without denotation. As Heisenberg sometimes uses meaningless in just this sense, we might inquire further if he thought that the simultaneous pair ( $p, x)$ was also without connotation. A logical adherence to the perturbation theory of measurement should lead to a rejection of the stronger statement. Heisenberg's intention is ambiguous; he seems, as in the passage we have just quoted, to deny even a connotation to a pair of simultaneous values ( $\mathrm{p}, \mathrm{x})$; but, in other places, he clearly implies that this is not so; for example, with reference to extrapolation into the past, he is ready to concede that it might be possible to calculate exact simultaneous values for past events 3 .

It is our view, however, that since the variables are also defined by reference to the measuring-process, the connotation is also lacking. However, a connotation can be lacking in one of two ways: either it is contradictory (i.e., nonsense), or it is indeterminate. For example, the actual values which specify the initial conditions of a classical system are indeterminate but not contradictory. An indeterminate case represents whatever is singular, unsystematic and irregular in a set of similar instances. Every law states only what is regularly and generally true. The statement that no deviation from the law occurs even in singular instances and unsystematically is a new law and not a corollary of the first. A minority of physicists, for example, among whom are Einstein, Popper, Bopp and Bohm 4, have held that the simultaneous pair $(\mathrm{p}, \mathrm{x})$ are determinate even if not always determinable. Margenau would hold that they are also determinable, although it would seem that an indefinite time-interval might be required for the simultaneous

1 Ibid., p. 15.

2 Ibid., pp. 20-46 where many examples of the Indeterminacy Relations are analysed.

3 Ibid., p. 20.

4 Albert Einstein: Philosopher-Scientist, pp. 81-87 where Einstein summarises his view of the quantum theory, as well as the account by Bohr of his discussion with Einstein on the foundations of quantum mechanics, ibid., pp. 199-242. Karl Popper, Logic of Scientific Discovery (London: 1959), chap. IX. F. Bopp. Observation and Interpretation (London: 1957), pp. 189-196. D. Bohm, Causality and Chance in Modern Physics (Princeton: 1957). Heisenberg lists some members of this school in Niels Bohr etc., pp. 12-29. Cf. infra, chap. v, Section IV. 
measurement 1. Suppes and Margenau have investigated the joint probability of non-commuting operators 2. Suppes has shown that in some cases at least, as, for example, in the first excited state of the harmonic oscillator, no joint probability distribution for $\mathrm{p}$ and $\mathrm{x}$ exists, while in other cases it does. Margenau has shown that in some cases negative probability values arise. Where no joint probability distribution exists, no formula exists to give sense to a simultaneous pair of values $(\mathrm{p}, \mathrm{x})$; i.e., it is non-sense and to this degree contradictory. However, where a joint probability exists, there is no contradiction in the formula ( $\mathrm{p}, \mathrm{x})$, even though the association of values has no determinate significance but only the indeterminate significance of a chance association governed by a joint probability distribution.

Having listed the three points on which we agree with Heisenberg's interpretation of the Indeternlinacy Relations, we now go on to mention the three points on which we find ourselves in disagreement. We disagree with the view (1) that the Indeterminacy Relations express a limitation of our knowledge of physical reality; (2) that a physical property is something other than what is defined in and through the measuring process itself, and (3) that there is no place for an objective (i.e., publicly objective) science of microphysical objects, except as a science of how we know and not of what we know. Since this last point is based upon an analysis of the measuring process in quantum mechanics, we shall devote the next section to a detailed discussion of this.

\section{SECTION III: THE QUANTUM THEORY OF MEASUREMENT}

\section{Three Stages of a Measurement}

The quantum theory of measurement as explained by Heisenberg describes the process in three stages: (a) before the interaction of instrument and object, (b) after the interaction, and, finally, (c) the act of observation. We shall consider each of these in turn.

(a) The isolated object before the measurement is said to be a pure case 3 , and the state is represented by a ray in abstract Hilbert space

1 H. Margenau, The Nature of Physical Reality (New York: 1950), p. 376.

2 P. Suppes, "Probability Concepts in Quantum Mechanics", Phil. Sci., XxviII (1961), pp. 378-389; H. Margenau, "Measurements and Quantum States", Phil. Sci., xxx (1963), pp. $138-157$.

3 A pure case (reiner Fall) or a pure state is one representable by a ray in Hilbert space; statistically it means that it is impossible to produce it by combining statistical ensembles with different characteristics. The term was introduced by $\mathrm{H}$. Weyl and used by Heisenberg and von Neumann. Cf., H. Weyl, Theory of Groups etc., p. 75;]. von Neumann, Mathematical 
which is usually taken to be a wave function $\Psi(\mathrm{x})$. The wave function is essentially related to a set of possible measuring processes or, as Heisenberg expresses it, it represents a potentiality which is actuated by a measuring process 1 . It is a pure case, and as such it denotes an individual something with properties some of which are precise and have definite numerical values, like rest mass, electric charge, etc., and others are imprecise but potentially precise since a precise value depends on the choice and subsequent performance of some measuring process. These potential properties occur in conjugate pairs. They are potential since exact values cannot be simultaneously assigned to both members of a pair of conjugate variables and, in the general case, no precise value need be assignable to either member of the pair. They are potential also with respect to the mathematical formalism, since value is obtained only by the mathematical transformation of the original pure case in which many values are potential into a new pure case which is the eigen state of one precise value.

The permanent precise properties of a system, like rest mass, electric charge, etc., are usually treated as invariance properties of the mathematical representation under some group of transformations. The potential properties are related to the mathematical elements of the transformation group 2. The wave function, then, represents something of general validity in itself and is - according to Heisenberg - objective; but since it does not represent a body or even a coherent set of events in space and time it is not fully objective... "Was wir mathematisch festlegen ist nur zum kleinen Teil 'objektives Faktum', zum grosseren Teil eine Uebersicht über Moglichkeiten", said Heisenberg 3.

Since a pure case is mathematically well-defined, it represents an ideal, abstract norm, which is a concept. This concept, moreover, has reference to a concrete individual system, since experimental evidence has shown that variables like energy, momentum, etc., are conserved in collisions between individual systems. The quantum description, in spite of the fact that it yields only ,statistical laws, intends to be a description of an individual system and not merely of

Foundations etc., pp. 306-307, 328-329; Heisenberg, Physical Principles etc., p. 56. The difference between a pure case and a mixture has been studied by E. P. Wigner in "The Problem of Measurement", Am. Jour Phys., XXXi (1963), p. 6, and by H. Margenau, Phil. Sci., xxx (1963), pp. 138-157.

1 W. Heisenberg, Niels Bohr etc., p. 27; Physics and Philosophy, pp. 4I, 53, 91, 180, 185; On Modern Physics (London: 1961), p. 9.

2 "All quantum numbers, with the exception of the so-called principal quantum number, are indices characterising representations of groups", H. Weyl, Theory of Groups etc. p. XXI.

3 Heisenberg, Dialectica, loco cit., p. 333. 
the properties of a collective. The pure case (or wave function), moreover, connotes a whole form, since the wave function which represents it, changes detenninistically and predictably, evolving through a perfectly definite series of wave functions, governed by the appropriate Schrodinger equation. It connotes then something which is formally one, whole and complete.

On the other hand, the only predictions made by the theory are statistical, and, consequently, the pure case is in some sense incomplete and imprecise. The pure case also describes a statistical ensemble of concrete cases, each characterised by the same wave function. There is a parallel between the "pure case" in quantum mechanics and the ('state" of a classical system. The theoretical representation of an individual system in classical physics is (as we have already noted) an idealised and abstract norm, of which actual concrete systems constitute a random sample. Classical physics deals with this ensemble by getting help from outside, viz., from a statistical "theory of errors" ; quantum mechanics on the other hand includes the statistical analysis within its own formalism. This, as we have already pointed out, is connected with the human way of scientific knowing.

Returning to the quantum theory of measurement: we are at a loss to know how to treat Heisenberg's view of the nature of the measuring process since he is not the author of the "standard" or " orthodox" view. However, it is generally held that "the standard view is an outgrowth of Heisenberg's paper in which the uncertainty relation was first formulated" 1 ; and it is clear from the brief defence Heisenberg made of it in 1955 that the regards it as the only authentic account 2. The first to explore the consequences of Heisenberg's ideas and to base a theory of measurement on them was von Neumann who published his classic work on the mathematical foundations of quantum mechanics in 1932 3. His view has come to be called the "orthodox" view of the Copenhagen School. The clearest summary of it, and the account from which we shall quote, is that given by London and Bauer 4 ,

Let the wave function before the measurement be denoted by $\Psi(x)$, and let $\Psi \mathrm{k}(\mathrm{X}), \mathrm{k}=1,2, \ldots$, be a complete set of eigen functions of

1 E. P. Wigner, Am. Jour. Phys., Xxxi (1963), p. 6.

2 Heisenberg, Niels Bohr etc., p. 27.

3 ]. von Neumann, Mathematische Grundlagen usw., translated under the title Mathematical Foundations 0/ Quantum Mechanics.

4 F. London and E. Bauer, La théorie de l'observation en mecanique quantique (Paris: Hermann, 1939). 
(say) the momentum $P$, where the corresponding eigen values of Pare $\mathrm{pk}, \mathrm{k}=1,2, \ldots$ Then $\Psi(\mathrm{x})$ can be written in the following way:

where

$$
\Psi(\mathrm{x})=\Sigma_{\mathrm{k}} \mathrm{a}_{\mathbf{k}} \Psi_{\mathbf{k}}(\mathrm{x})
$$

This is a pure case.

$$
\sum_{\mathbf{k}}\left|\mathrm{a}_{\mathbf{k}}\right|^{2}=\mathrm{I}
$$

The process of measurement itself takes place in two stages: (b) the interaction between the object and the apparatus which is represented mathematically by the transformation of the pure case $\Psi$ into a mixture of the states $\Psi \mathrm{k}(\mathrm{X})$, and (c) an act of observation which "registers" which of the states $\Psi \mathrm{k}(\mathrm{X})$ has been "actualised" by the interaction.

\section{Formation oj a Mixture}

Let us consider first of all the interactions between the object and the apparatus; and let the property measured by the apparatus be the momentum P. The object-plus-apparatus comprises a closed and isolated macroscopic physical system which is subject to the laws of physics. Assuming that the quantum theory applies also to macroscopic systems, it will have a comprehensive wave function $X$ in which both the variables of the object, viz., $x$, p, etc., and the variables of the apparatus, viz., y, q, etc., will be present. Let the variable which is correlated with the measured property $\mathrm{P}$ of the object be $\mathrm{z}$. Let zo, $z_{1}, \ldots \mathrm{Zi}, \ldots$ be its eigen values (they are, say, the positions of a pointer on a scale) and let $\Phi_{0}(\mathrm{y}), \Phi_{1}(\mathrm{y}), \ldots \Phi_{\mathrm{i}}(\mathrm{y}), \ldots$ be the corresponding eigen functions of the apparatus. Then, the nature of a measuring apparatus is that there should exist such a correspondence between the states $\Psi \mathrm{k}(\mathrm{X})$ of the object and the states $\Phi_{\mathrm{k}}(\mathrm{y})$ of the apparatus, that from the pointer reading $\mathrm{Zk}$ of the apparatus, the value $\mathrm{Pk}$ for the momentum of the object can be inferred.

Before the interaction, the comprehensive wave function $\mathrm{X}$ was simply the product of the wave function $\Psi$ of the object and of the wave function $\Phi_{0}$ of the zero state of the apparatus: Le.,

$$
\mathrm{X}=\Psi \Phi_{0}=\Sigma_{\mathrm{k}} \mathrm{a}_{\mathrm{k}} \Psi_{\mathrm{k}} \Phi_{0}
$$

After the interaction, the only form which the comprehensive wave function $x($ final $)=X I$ can have, and which is in keeping with the nature and function of the measuring-process is,

$$
X I=\Sigma_{\mathbf{k}} \mathbf{a}_{\mathbf{k}} \Psi_{\mathbf{k}} \Phi_{\mathbf{k}}
$$


That is, to every $\Psi \mathrm{k}$ there is coupled a $\Phi_{k}$, or in other words, from every potential value $\mathrm{Zk}$ of the apparatus, we can infer a corresponding value $\mathrm{Pk}$ for the momentum of the object. The final state $\chi_{\boldsymbol{p}}$ of the combined instrument and object is a pure case as long as they constitute an isolated system and, as long as this is true, the values $\mathrm{Zk}$ are only potential in the wave function, for it represents the total system.

A measurement, however, does not consider the total combined system, but only one part of it, viz., the apparatus. Examining the total wave function $\chi_{p}$ for the information it can yield about the state of the apparatus, it can be shown that this is represented by what is called a mixture of the eigen states $\Phi_{\mathbf{k}}$ present in Xr. Another mixture, but this time of the $\Psi_{k}$ is in one-to-one correspondence with this and represents the condition of the object. A mixture is a virtual ensemble of different pure cases, each present with a certain determinate probability; here the probability associated with $\Phi_{\mathrm{k}}$ and with $\Psi \mathrm{k}$ is lakl ${ }^{2}$. That is, from the point of view of the apparatus, the original pure case $\Psi$ of the object is transformed into a mixture containing all the eigen states present in $\Psi$, each with its detemiinate probability now actuated by the interaction. Such a mixture is an ordinary Gibb's ensemble like those used in classical statistical mechanics 1. The state of the system is now determinate but still unknown. The situation might be compared with a card drawn at random from a pack of cards, in which each card is marked with one of the $\Psi \mathrm{k}$ and each $\Psi \mathrm{k}$ is represented in the pack with a frequency proportional to lakl ${ }^{2}$. The ideal frequency of a set of random drawings from the pack is predicted by the theory, but what the result will be in any concrete case cannot be inferred from it. At this stage of the measurement, the quantum mechanical situation would be like a card drawn from such a pack, lying face down and not yet scrutinized. The quantum mechanical case has by now been transformed into a case of classical statistics.

\section{Act ot Observation}

The measurement is completed by an act of observation which ascertains which of the pointer values $\mathrm{Zk}$ has been actuated by the interaction. From a pointer value $\mathrm{Zk}$, one concludes that the object,

\footnotetext{
1 The reduction of a pure case to a mixture is often described as a projection of the pure case on to its eigenstates; for the pure case is represented by a ray in Hilbert space which is spanned by a complete set of eigenstates as if each of these were a coordinate axis in the Hilbert space. The reduction of a pure case to a mixture is its projection on to the "coordinate axes" of the space; the probability that a projection will take place along any particular axis is proportional to the squared length of its projection on this axis. For this reason, the measuring process is often called a projection operator.
} 
immediately after the measurement, is in the pure state $\Psi_{k}$. Thus, the final act of observation is a process of sampling the mixture (which is a probability distribution) and of registering the contingent factual outcome.

One fact, however, should be noted; that the comprehensive wave function $\chi_{f}$ for the combined object-apparatus system considered in isolation from its surrounding contains more information than do the separate mixtures produced by the interaction. Certain correlations between the states of the object and those of the apparatus - viz., superposition states - have been destroyed by the measurement 1 . This results in an increase in entropy of the entire system consequent upon the act of observation 2.

Apart fron1 its more subtle and complicated character, which distinguishes it from the theory of measurement in classical physics, the quantum mechanical theory of measuren1ent seems to be straightforward enough and obscure philosophical questions seem to be fairly remote. However, just as the initial insight of Heisenberg into the foundations of physics was fraught with philosophical consequences, so the defects in his philosophical view came to be incorporated into the very heart of quantum mechanics, viz., into the theory of measurement.

\section{The Observer in Quantum Mechanics}

According to Heisenberg, the function of the observer is to "register decisions" 3 , i.e., to record which of the possibilities contained in the statistical mixture described above has in fact been actualised by the measurement. He says that the recording can be done as well by a photographic-plate as by a human observer. However, as Wigner and others have shown, this does not follow from the theory, since in so far as the object-plus-instrument-plus-photographic-plate constitute a larger isolated system, the theory allows one to deduce no more than the pure case. To obtain verifiable formulae, one must pass to the next stage, namely, of the formation of a mixture, and this supposes that the system is subject to a super act of observation from outside which interferes with the state of the system. To go from the pure state to

\footnotetext{
1 "Of paramount philosophical significance... is that (3) the whole is always more, is capable of a much greater variety of wave states than the combination of the parts. Disjoint parts in an isolated system of fixed wave states are in general not statistically independent even if they do not interact", H. Weyl, Philosophy of Math. etc., p. 263; d. also, London and Bauer, loco cit., pp. 34-37.

2 London and Bauer, loco cit., p. 30; also von Neumann, loco cit., pp. 379-398. Cf. also Appendix, pp. 180-2.

3 Heisenberg, Niels Bohr etc., p. 22.
} 
the mixture, and thence to the question of fact, a union must take place between the photographic-plate and the sensibility of a human observer. While this union is physically no different from that between object and apparatus, the human sensibility, however, has the "characteristic and familiar power which we can call the 'power of introspection'" 1 by which it can take cognizance of its own state, and so emerge from the indeterminacy of a mixture to the determinacy of fact by an act of auto-observation 2. Fron1 a knowledge of his own state, the human observer infers the correlative state in which the object finds itself after the measurement. The process of passing from the initial pure state of the object to the final pure state after observation is called the "reduction of the wave packet". It is physical as we have already explained; it is psychological since it requires the intervention of a human act of auto-observation, and it is also logical because, in the language of complementarity, the wave picture dissolves into that of the complementary particle picture, and this fact gives its name to the entire process, viz., the "reduction" or "contraction of the wave packet".

\section{Reduction of the Wave Packet}

One of the most controversial topics in quantum mechanics to-day is the reduction of the wave packet. There are three problems. (1) Does the reduction entail a real occurrence in the physical object independent of the conscious act of observation; or is it merely a "reduction of knowledge", i.e., a change in representation due to the acquisition of new information about the object without entailing a significant change in the object; or does it include both of these? This will be discussed in the next chapter. (2) Is the Projection Postulate a necessary part of quantum mechanics? That is: is a definite eigen state the new pure state produced by the act of measurement or does the act of measurement measure the state as it was before the measurement, whatever happens to the system after or as a result of the measurement (e.g., the system might be destroyed by the measurement as, for example, when a photon is absorbed)? This is principally a physical

1 London and Bauer, loco cit., p. 42.

2 Note how the act of auto-observation, as described by London and Bauer, assumes a coincidence or at least a parallelism between consciousness (Le., the content of the conscious act of observation) and the physical substratum (Le., the physical state of the eyes, nerves, brain, etc., ot the human observer). Implied in this account is also the theory that consciousness (or rather acts of observation of reality) also follows quantum mechanical laws. We shall return to this later in chap. v. 
problem and it will not be discussed in this book 1. (3) Is the "reduction of the wave packet" a process essentially different from the mere sampling of a statistical distribution, whether this be a classical (stochastic) distribution or one of some non-classical type? This problem will be discussed in the section entitled "Formal Objectivity" in the next chapter and in chapter VI.

Heisenberg, von Neumann, Wigner, London and Bauer regard the reduction of the wave packet as a new and unique kind of psychophysical proiection operator terminating in the projection on to the plane of actuality of one of the potential states represented in the wave packet. Many physicists find this explanation unclear and permeated with dubious epistemological presuppositions. Landé complains: "No agreement has been reached whether the said 'contraction' is physical, mental, real, pictorial, objective or subjective. But something must contract, since Heisenberg said so thirty years ago $" 2$.

\section{Obiectivity of Quantum Mechanics}

A disturbing question is suggested by the views of Heisenberg, Wigner, von Neumann and others, that the (private) sensibility of the individual human observer is an essential determinant of the object of quantum mechanics. If this is so, how can public objectivity, a necessary condition of all science, exist in quantum mechanics?

One answer is that given by London and Bauer 3. The instrument and the eye are macroscopic systems. Hence, the quantum mechanical treatment of the link between the two must approach the classical limit, which is, of course, the paragon of public objectivity. They argue that the coupling between the eye and the apparatus changes the apparatus only negligibly and that, consequently, the same correspondence exists between the apparatus and the eye of any observer 4 .

1 Among those physicists who reject the Projection Postulate are, Margenau, Lande, Feyerabend, Schrödinger. Cf. H. Margenau, Phil. Sci., xxx (1963), I-I6, 138-157; P. K. Feyerabend, Frontiers of Science and Philosophy; A. Lande, From Dualism to Unity in Quantum Mechanics (Cambridge: 1960); E. Schrödinger, Naturwiss., XXIII (1935), p. 812. 2 A. Lande, "From Dualism to Unity in Quantum Mechanics", in Current Issues in the Philosophy of Science, ed. by H. Feigl and G. Maxwell (New York: 1961), p. 355.

3 London and Bauer, loco cit. pp. 48-51; also D. Bohm, Quantum Theory (New York: Prentice-Hall, 1951); G. Ludwig, Die Grundlagen der Quantenmechanik (Berlin: 1954); P. K. Feyerabend, Observation and Interpretation (London: 1957); A. Daneri, A. Loinger, G. M. Prosperi, Nucl. Phys., XxxiII (1962), p. 297.

4 Recent studies have shown that the size of the apparatus is of considerable importance to the measurement. E. Wigner and H. Salecker showed the necessity of relatively massive apparatus for the precise determination of time (Phys. Rev., CIX, 1958, p. 571); for the influence of the size of the apparatus on the accuracy of measurements, d., E. Wigner, 
London and Bauer conclude their study with the reassuring statement: "The possibility of prescinding from the individuality of the observer and of creating a collective scientific consciousness cannot be seriously questioned" 1 .

This answer is based upon the Correspondence Principle and upon the assumption that quantum mechanics, in so far as it is applicable to macro-phenomena, gives nothing more that what classical physics would give in these cases. This is a common view of the Correspondence Principle, and not altogether a correct one; for quantum physics could give classical results in certain appropriate limiting cases without excluding the possibility that quantum physics contains something more, for example, a more exact explanation of the relation between observer-subject and observed-object in physics - even of macroscopic phenomena. Heisenberg, Wigner, von Neumann, for example, clearly imply that something more is given 2. Others, like Ludwig, try to avoid this conclusion by restricting the applicability of quantum physics to microscopic phenomena and to marginal cases 3 . The majority of physicists, however, among whom is Heisenberg, hold that the quantum mechanical domain includes also the domain of classical physics. There is a connection between this view and the insistence on the inescapable precence of subjectivity in modern physics.

\section{Summary}

The philosophy of complementarity, while successful in providing physicists with a common language with which to describe quantum phenomena, also contains a theory about scientific method and about human knowing which is open to criticism. In this chapter, we criticised the following points arising out of the philosophy of complementarity: psycho-physical parallelism; the view that quantum mechanical properties are to be defined classically; and the perturbation theory

Zeit f. Physik, Cxxxi (1952) p. 101; Amer. Jour. Phys., xxxi (1963), p. 6; H. Araki and M. Yanase, "Measurement of Quantum Mechanical Operators", Phys. Rev., cxx (1960), pp. 622-626; M. Yanase, "Optimal Measuring Apparatus", Phys. Rev., CXXiII (1961), pp. 666-668. Wigner concludes: "This raises the suspicion that the macroscopic nature of the apparatus is necessary in principle" (Am. Jour. Phys., XXXI, 1963, p. 6).

1 London and Bauer, lac. cit., p. 49.

2 E. Wigner, "Remarks on the Mind-Body Problem", in The Scientist Speculates, ed. by I. J. Good (London: 1962), pp. 284-301; ]. von Neumann, Mathematical Foundations etc.; W. Heisenberg, Niels Bohr etc., pp. 12-29.

3 G. Ludwig, "Gelöste und ungeloste Probleme des Messprozesses", in Werner Heisenberg und die Phvsik unserer Zeit, ed. by F. Bopp (Braunschweig: 1961), pp. 150-181. Ludwig 
of measurement. In the course of the criticism, we elaborated the distinction between two types of concepts with different logical structures; viz., operational or observational concepts which state a similarity between things judged on the basis of appearance or utility to $u s$, and explanatory concepts which state a similarity between things judged on the basis of a self-defining set of different relations between things. We have shown how a physical concept is definable by any appropriate measuring-process. The description of the measuringprocess and, hence, the definition of the physical property involve the two classes of concepts described above, but in different ways. This leads us to regard the Indeterminacy Relations, not as stating limitations of our knowledge, but as describing more exactly the behaviour of individual systems. 